\title{
LA MÉDIATION INSTITUTIONNELLE : UN MODE NOUVEAU DE LÉGITIMATION D'UNE ACTION PUBLIQUE EN MUTATION
}

\author{
Elisabeth Volckrick et Stéphanie Rigo ${ }^{1}$ \\ À Pierre-Yves Monette, Bernard Hubeau, \\ Caroline Cosyns, Jean-Marie Hannesse \\ et Philippe de Surä̈
}

L'institution du médiateur dans la fonction publique ou ombudsman, «celui qui parle pour autrui $»^{2}$, est présente aujourd'hui dans une centaine de pays. En Belgique, elle est née au lendemain du vote protestataire du «dimanche noir $»^{3}$ en novembre 1991, et dans la foulée du mouvement citoyen de la «marche blanche » de 1996. Ce fut une prise de conscience par le politique de l'ampleur du fossé existant entre les citoyens et les institutions et du danger qu'il constitue pour la démo-

1 Elisabeth Volckrick est professeur invité au Département de communication de l'Université catholique de Louvain (marie-elisabeth.volckrick@uclouvain.be). Stephanie Rigo y est assistante.

2 Selon les pays, on l'appelle aussi Protecteur du citoyen, Commissaire aux Droits de l'Homme, Commissaire à la Bonne administration, Avocat du peuple, Défenseur du peuple, Vigie de la Nation, etc.

3 «Dimanche noir» ou «Zwarte zondag » est le terme utilisé, en Belgique, pour désigner une poussée électorale d'extrême droite. La première eut lieu aux législatives du 24 novembre 1991 avec le demi-million de voix obtenues par le Vlaams Block (aujourd'hui Vlaams Belang.).

Recherches en communication, $\mathrm{n}^{\circ} 25$ (2006). 
cratie. La médiation institutionnelle s'est depuis développée aux différents niveaux : fédéral, régional, communautaire et local. Elle témoigne à la fois de tensions, de dysfonctionnements, de malaises ressentis dans l'action publique en général et d'un souhait d'y remédier en créant des espaces de communication, de discussion et de négociation instituée entre l'administré et l'Etat.

L'objectif de cet article est de comprendre la nature des processus de légitimation mis en ouvre par les pouvoirs publics au travers des pratiques de médiation. Autrement dit, les pratiques des médiateurs institutionnels révèlent-elles la nature des rapports sociaux que tentent de légitimer l'administration? Comment les médiateurs gèrent-ils les différents problèmes et tensions au centre desquels ils se situent en tiers ? Comment tentent-ils de créer du consensus ? On peut aisément prévoir la délicatesse et la complexité de la situation. Les médiateurs institutionnels sont, d'une part, plongés au cœur de différentes formes

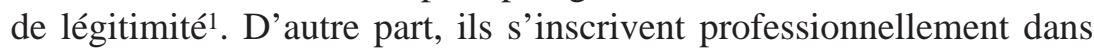
une relation de pouvoir et d'autorité qui est au moins triple: ils se retrouvent dans un rapport de légitimité avec l'autorité dont ils dépendent, avec les administrations et avec les administrés.

Il existe bien sûr différents modes d'approche de la médiation institutionnelle. Nous soutiendrons l'hypothèse que la médiation institutionnelle constitue un processus de légitimation nouveau dont la dimension communicationnelle est centrale et qui témoigne d'un nouveau mode d'action de l'Etat.

Pour répondre à nos questions, nous avons entrepris plusieurs démarches. Nous avons rencontré à plusieurs reprises un groupe de cinq médiateurs ${ }^{2}$ de la fonction publique. Nous leur avons demandé

1 Un des grands intérêts de cette fonction est d'être justement au cœur de différentes légitimités et de chercher à préserver cette pluralité. VOLCKRICK E., «Médiation institutionnelle et légitimité délibérative », Chroniques de Droit Public, Publiekrechetijke Kronieken, Dossier: Pouvoir politique et médiation institutionnelle, De relatie tussen politiek en de ombudsman, $\mathrm{n}^{\circ} 2$ 2, 2006. p. 518524.

2 Un groupe de recherche "Légitimité et médiation institutionnelle » a été creé dans le cadre du séminaire scientifique du LASCO (Laboratoire d'analyse des systèmes de communication d'organisation) du Département de communication de l'UCL. Ont participé à ses discussions, les médiateurs : Pierre-Yves MONETTE, Bernard 
de mettre en récit des situations problématiques qu'ils avaient concrètement rencontrées et qui touchaient à des questions de légitimité, et de les analyser, avec notre accompagnement. Devant la complexité de certaines questions soulevées, nous avons également organisé, toujours en collaboration avec les médiateurs, une journée d'étude ${ }^{1}$ sur le thème « Pouvoir politique et médiation institutionnelle», réunissant des médiateurs institutionnels, des hommes politiques, des communicatologues, des sociologues, des politologues, des juristes, des philosophes. Dans les deux langues nationales, la médiation institutionnelle a été mise en question et analysée. Nous avons, par ailleurs, pris connaissance des rapports annuels de nombreux médiateurs institutionnels ${ }^{2}$.

L'institution du médiateur de la fonction publique, basée sur un concept scandinave, est une autorité publique mais totalement indépendante du gouvernement, du Parlement et de la Justice. Elle est souvent présentée dans ses aspects purement fonctionnels. Dans cette perspective, la fonction du médiateur institutionnel viserait à recevoir les plaintes des citoyens, à concilier les points de vue de deux ou plusieurs parties en différend, voire en conflit, en l'occurrence un administré et une administration (internationale, fédérale, régionale, communautaire ou locale) et, à partir d'une connaissance de première main des plaintes et des dysfonctionnements, à formuler des recommandations et à conseiller le parlement et le gouvernement, dans le cas du médiateur parlementaire ou, plus généralement le décideur, dans le cas du médiateur administratif. Dans cette acception, la médiation institution-

HUBEAU, Caroline COSYNS, Philippe de SURAŸ, Jean Marie HANNESSE, et les chercheurs de l'UCL: Stéphanie RIGO, Elisabeth VOLCKRICK et André BERTEN.

1 La journée d'études «Médiation institutionnelle et pouvoir politique » s'est déroulée le 18 novembre 2004 à Louvain-la-Neuve. Cette journée, organisée à l'initiative du Département de communication de l'UCL et du CRIO (Centre de recherche interdisciplinaire sur l'Ombudsman), a réuni des médiateurs dont Bernard HUBEAU, Pierre-Yves MONETTE, Jean-Luc LABBE, Rita PASSEMIERS ; des politiques dont Trees MERCKX, Jean-Pierre MALMENDIER ; des analystes dont Kris DESCHOUWER, Christine GUY-ECABERT, Jean-Louis GENARD, Raphaël GELY, Christian de VISSCHER, Jean DE MUNCK, André BERTEN et Elisabeth VOLCKRICK.

2 L'importante quantité d'informations et de documentations recueillies et reçues nécessiterait incontestablement d'autres études qui dépassent le cadre du présent travail. 
nelle apparait comme une ressource de légitimation des processus décisionnels et du fonctionnement de l'action administrative et le médiateur institutionnel comme un rouage supplémentaire souple visant à améliorer le fonctionnement d'un système administratif de plus en plus complexe et, par là, à en restaurer une certaine légitimité.

Cette présentation de la médiation institutionnelle n'est certes pas dénuée de vraisemblance. La médiation vise à l'amélioration du fonctionnement administratif et a une fonction de légitimation de l'action administrative. Cependant, notre recherche nous a montré que l'on ne peut, en aucun cas, réduire la médiation institutionnelle à cette dimension fonctionnelle. Il s'y joue d'autres enjeux importants.

Pour bien comprendre la nature des processus de légitimation mis en œuvre par les pouvoirs publics au travers des pratiques de médiation, nous avons été amenée à les replacer dans le contexte plus général des transformations de l'action administrative. De nombreuses études ${ }^{1}$ ont souligné les tensions qui traversent l'administration. Aucune politique publique ne peut plus désormais éviter la question complexe de l'articulation de contraintes ${ }^{2}$ d'efficacité et de légitimité.

Saisir la signification de l'apparition de mécanismes institutionnels tels la médiation sera donc notre premier point. Nous montrerons que la médiation institutionnelle témoigne d'une insuffisance des mécanismes institutionnels classiques et apparaît comme un nouveau mode de légitimation de l'action publique. Parallèlement à ce contexte empirique, il faut aussi - et ce sera notre second point - en comprendre le contexte théorique. Introduire une perspective normative fera apparaitre l'importance de l'usage communicationnel du langage dans les processus de légitimation. Nous expliciterons alors ce qui constitue la spécificité de la médiation institutionnelle.

1 EWALD Fr., L'Etat providence, Paris, Grasset, 1986; GENARD J.-L., « Spécificités de l'administration publique belge et reforme administrative », in GOBIN C. et B. RIHOUX (eds.), La démocratie dans tous ses états, Système politique, entre crise et renouveau, Louvain-la-Neuve, Académia Bruylant, 2000, pp. 163-174; VRANKEN D., Les métamorphoses de l'administration, Loverval, Quartier libre, Labor, 2006.

2 Florence Carion et John Cultiaux constatent une double contrainte assez semblable dans le monde de l'action humanitaire. Voir « Le communicateur dans le mouvement de professionnalisation de l'action humanitaire », dans ce volume. 


\section{La légitimité de l'action administrative en mutation}

Afin de situer l'émergence de la médiation institutionnelle et d'en comprendre les enjeux, il convient de se rappeler que la fonction d'administrer s'est modifiée fortement au cours du temps. Elle est aujourd'hui traversée par des logiques d'action extrêmement différentes, voire contradictoires. Les critères possibles de la « validité » de l'action administrative sont devenus eux aussi plus complexes.

En Belgique, jusqu'au dernier quart du XIXème, la fonction d'administrer semble avoir été dominée par un modèle de type libéral, principalement dévolu à encadrer un marché libre par des règles de droit privé. Ce que l'on a appelé l'Etat social s'est progressivement développé, sous la poussée du socialisme et du progressisme social, en réaction aux déséquilibres engendrés par l'Etat libéral. L'Etat n'est plus seulement gardien des libertés, il se fixe aussi des objectifs dans le domaine social, éducatif, sanitaire et de planification. Cette extension des tâches étatiques transforme les attentes du citoyen désormais en position de demandeur de prestations de la part d'un Etat dit providence. Parallèlement, on observe un décentrement accentué de l'Etat vers le pouvoir exécutif. L'administration publique connaît une importante croissance, devient imposante, bureaucratique, spécialisée, cloisonnée. Les ministères et leurs nombreux fonctionnaires ne servent plus seulement à «appliquer des lois », l'action publique qu'ils mènent est devenue très complexe.

On doit à Max Weber ${ }^{1}$ une remarquable description de l'ensemble de la gestion administrative. Il a montré combien elle reposait sur la forme de légitimité que donne «la croyance en la légalité des règlements arrêtés et du droit de donner des directives qu'ont ceux qui sont appelés à exercer la domination par ces moyens ». A la fin de sa vie, cette forme de rationalité formelle, très présente à son époque, était en conflit avec une forme de rationalité matérielle caractérisée par l'exigence d'une prise en compte par l'Etat de valeurs substantielles (égalité, justice, liberté, ...). Weber voyait dans ce conflit une des difficultés centrales de la modernisation des sociétés contemporaines. La logique d'application des règles cédait progressivement la place à une logique

1 WEBER M., (posthume 1921), Economie et société, tome I, Paris, Plon, 1971. 
« fonctionnelle » visant la réalisation d'objectifs. Les critères de validité de l'action administrative se transformaient et passaient de la conformité à la règle (loi, arrêtés, directives, règlements,...) à l'efficacité de l'action en fonction d'objectifs généraux centralisés. L'administration, traversée par ces logiques différentes, connut une tension qui ne cessa de s'amplifier durant le développement de l'Etat social.

A partir des années 70, apparaissent de nouvelles tensions. Le modèle de l'Etat social est en «crise » et fait l'objet de deux critiques distinctes ${ }^{1}$. Une première critique dénonce «les effets pervers d'une intervention accrue du pouvoir administratif sur la personnalité, sur la solidarité sociale ou sur le développement culturel ». On se souvient de l'œuvre de Michel Foucault qui a montré combien les appareils disciplinaires et le pouvoir normalisateur stigmatisent le destin d'une société de plus en plus rationalisée. Une seconde critique est la critique néolibérale de l'Etat social qui dénonce «les effets d'irrationalité liés à l'interventionnisme croissant de l'Etat».

C'est dans ce contexte qu'en Belgique, l'Etat social connaît de profonds changements. La création des communautés et des régions amène une nouvelle structuration de l'Etat. Son administration, en même temps qu'elle se cherche à travers la décentralisation, est confrontée à l'impératif de «modernisation » que l'on rencontre dans tous les pays occidentaux. De nouvelles méthodes de gestion se développent. A travers ces changements, la visée de réalisation d'objectifs se maintient mais avec une exigence d'efficacité plus grande. Pour être efficients et atteindre ses objectifs, l'administration a besoin de la participation effective et directe de son public à la politique qu'elle mène. Pour cela, les services publics ont intérêt à faire preuve de flexibilité et à susciter la mobilisation du public auxquels ils s'adressent. En pleine revendication identitaire, ce public n'accepte plus une administration pour laquelle il n'est qu'un dossier, un numéro, et qui tente de le placer dans ses catégories préétablies qui ne correspondent plus à la complexité de la réalité.

Toujours subordonnée à l'autorité du pouvoir politique, l'administration quitte une logique purement verticale où elle n'était consi-

1 LENOBLE J. et A. BERTEN, «L'espace public comme procédure », in Raisons pratiques, Pouvoir et légitimité. Figures de l'espace public, Paris, Editions de l’EHESS n³, 1992, p. 84. 
dérée que comme une instance d'application et de contrôle des décisions publiques et voit sa sphère d'action s'accroître considérablement. Il lui faut de plus en plus écouter, conseiller, coordonner, renvoyer les informations, communiquer c'est-à-dire créer du consensus et négocier tant en amont qu'en aval des décisions publiques. Face à des situations concrètes, variées, complexes, plurielles qu'elle rencontre, l'administration est appelée à s'adapter aux situations, à les connaître en particulier, à réviser, à modifier la formulation même de la loi abstraite et générale, sèche et impersonnelle. Ces transformations modifient fortement la relation de l'Etat, de l'administration et du citoyen.

La question de la légitimité de l'action administrative se pose alors d'une façon nouvelle. Dans le modèle formel-légal, l'administration tire ultimement sa légitimité du pouvoir législatif qui choisit le pouvoir exécutif qui commande à l'administration. L'action de l'administration a donc une légitimité dérivée. Mais la quotidienneté du travail avec les publics concernés fait surgir la question d'une légitimité directe de l'action administrative. Il s'ensuit que les processus de légitimation traditionnels ne sont plus pertinents sur ce terrain où des contraintes d'efficacité et de légitimité doivent nécessairement co-exister. «En plus de la conformité aux règles légales et de l'ajustement aux objectifs, il y a un troisième critère possible de la « validité » de l'action administrative : son efficacité dans la construction d'un consensus politique $» .{ }^{1}$ Comment l'administration, toujours sous l'autorité du politique, va-t-elle penser ce nouveau rapport au public ? La redéfinition de sa mission n'est pas une mince affaire et la plonge dans un champ de contradictions et de conflits. Les problèmes d'action publique qu'elle rencontre ne sont plus seulement des problèmes endogènes, ils proviennent aussi de l'extérieur. Les citoyens et autres acteurs extérieurs interpellent l'administration et les problèmes qu'ils posent génèrent des effets de rétroactivité sur l'administration qui est amenée à penser ses problèmes internes sur un mode plus réflexif². C'est dans ce contexte qu'il

1 OFFE C., Disorganised Capitalism, Cambridge, Polity Press, 1985, p. 308, cité par DE MUNCK J., «Plus qu'un conseil et moins qu'un ordre », Chroniques de Droit Public, Publiekrechetijke Kronieken, Dossier : Pouvoir politique et médiation institutionnelle, De relatie tussen politiek en de ombudsman, $n^{\circ} 2$, 2006. p. 525530.

2 Toutes ces évolutions ne signifient pas une reconversion totale de l'administration et de sa gouvernance. Nombre d'agents et de services continuent à exercer une fonction d'exécution plus traditionnelle. 
faut situer l'apparition de mécanismes institutionnels tels la concertation et la médiation dans le traitement des problèmes collectifs ou individuels. On assiste, comme le soulignent André Berten et Jacques Lenoble, à « une tendance croissante de nos sociétés à associer de manière plus étroite les différents groupes de la société civile à la régulation de l'économie ou d'autres fonctions sociales. Ce qui témoignerait d'une insuffisance des mécanismes institutionnels classiques mis en place par la démocratie libérale du XIXème siècle, ou par l'Etat social du XXème, pour assurer la régulation sociale $»^{1}$.

La prise en compte de l'évolution de l'administration et de ses tensions permet de situer l'institution du médiateur institutionnel comme une des tentatives imaginées pour solutionner ce problème de double exigence d'efficacité et de légitimité, de façon régulée et systématique $^{2}$. Elle permet aussi de voir que la médiation institutionnelle intervient sur le terrain même de la légitimation, comme nouveau mode d'action de l'Etat ${ }^{3}$. La médiation institutionnelle n'est pas seulement un dispositif de gestion de plaintes, elle est un nouveau mode de légitimation de l'action publique dont la spécificité réside essentiellement dans la négociation et la création de consensus. Elle se présente, et nous y reviendrons plus loin, comme un mode de légitimation par le bas de la démocratie. C'est ce qui échappe à ceux qui la considèrent uniquement comme une technique efficace de résolution de problèmes, de gestion de plaintes et de facilitation ou qui s'obstinent à voir le médiateur institutionnel comme un simple organe de la loi.

Communiquer, au sens d'interagir et de créer du consensus, apparaît aujourd'hui comme un important facteur de légitimation.

1 LENOBLE J. et A. BERTEN, op.cit. p. 85.

2 DE MUNCK J., « Plus qu'un conseil et moins qu'un ordre », Chroniques de Droit Public, Publiekrechetijke Kronieken, Dossier: Pouvoir politique et médiation institutionnelle, De relatie tussen politiek en de ombudsman, $n^{\circ} 2$, 2006. p. 525530.

3 Cf. notamment GUY-ECABERT Ch., «Le médiateur institutionnel dans la perspective des nouvelles formes d'action de l'Etat: l'exemple du Collège des médiateurs fédéraux », Chroniques de Droit Public, Publiekrechetijke Kronieken, Dossier : Pouvoir politique et médiation institutionnelle, De relatie tussen politiek en de ombudsman, $\mathrm{n}^{\circ}$ 4, 2005. p. 885-890 ; FAGET J., « Médiation et post-modernité. Légitimation ou transformation de l'action publique ? , Négociations, Bruxelles, De Boeck Université, n², 2006, p. 51-62. 


\section{Communication et légitimation}

Parallèlement à ce constat empirique marqué par la transformation progressive des formes de l'action publique, on constate un renouveau marqué du contexte théorique. Ainsi, la réflexion sur la régulation sociale et sur la démocratie fait à nouveau recette et provoque de nombreux débats. On discute d'une « nouvelle figure, encore inachevée, d'une démocratie redéfinie $»^{1}$, de l'émergence d'une reformulation de la grammaire du lien social ${ }^{2}$. Notre propos n'est pas de reprendre ici ces débats. Disons que sur ceux-ci s'articulent des perspectives nouvelles ${ }^{3}$ qui intègrent des éléments de différents courants philosophiques et sociologiques. Dans ce contexte, nous devons nous demander quel cadre conceptuel choisir pour réactualiser une réflexion sur les notions de légitimité et de légitimation. Après avoir rappelé très brièvement quelques aspects de l'approche sociologique, nous montrerons que l'idée de légitimité s'enracine dans une caractéristique des processus cognitifs : la normativité.

L'expérience empirique dans tous les domaines de relations entre hommes fait apparaître que tout pouvoir se veut légitime. "Tout pouvoir veut être fondé en raison et entraîner de ce fait une adhésion unanime (acceptation et intériorisation des critères estimés rationnels)» ${ }^{4}$. Pourquoi ? Parce que seule la légitimité lui confère la durée et l'adhésion. Weber distingue la puissance ou le pouvoir - possibilité de contraindre le comportement d'autrui à sa propre volonté - de la domination - croyance dans la légitimité d'un ordre reçu.

Selon une perspective sociologique ${ }^{5}$, le concept de légitimation vise à décrire un processus - et ses effets - par lequel des individus sont amenés à reconnaître la légitimité du pouvoir, des institutions,

1 LENOBLE J. et A. BERTEN, idem. Les auteurs présentent les débats qui ont structuré la philosophie politique des vingt dernières années et, notamment, les travaux de J. Rawls, J. Habermas, N. Luhmann.

2 Cf. notamment DE MUNCK J. et M. VERHOEVEN (éds), Les mutations du rapport à la norme. Un changement dans la modernité ?, Bruxelles, De Boeck, 1997.

3 Cf. notamment les travaux de J-M. FERRY, J-P. DUPUY, L. BOLTANSKI et L. THEVENOT.

4 ENRIQUEZ E., Les figures du maître, Paris, Arcantere, 1991. p. 30.

5 Nous renvoyons à l'article de G. LIENARD, « Métiers de la communication et légitimation : approche sociologique », dans ce volume. 
des comportements, des usages, des conventions, des discours, etc. La dimension symbolique est constitutive de l'individu et de la société. Les grilles symboliques de catégorisation, de perception, d'évaluation coordonnent les perceptions du monde social et constituent les mécanismes sous-jacents à la reconnaissance de la légitimité. De tels mécanismes permettent de rendre compte de l'effet de l'intériorisation de la domination par les dominés. Ils donnent à penser que la légitimation pourrait se limiter à la validation et à l'occultation de la domination. La question se pose alors de savoir s'il pourrait y avoir une forme de légitimité qui ne soit pas trompeuse ou «idéologique».

Mais nous ne pouvons en rester là. Comme le fait remarquer Berten ${ }^{1}$, la perspective sociologique ne pose pas la question de savoir si, en fin de compte, il y a ou il n'y a pas de justification ${ }^{2}$ normative à ce qui est reconnu comme légitime. Elle ne rend pas compte de façon satisfaisante de la normativité inhérente aux comportements humains : tout comportement quel qu'il soit est un comportement qui implique une certaine forme de norme. Elle ne rend pas compte non plus de ce qui est impliqué dans l'usage discursif du langage : le langage est intrinsèquement normatif. L'idée de légitimité perd son sens véritable si est évacuée sa dimension cognitive spécifique, celle d'une normativité qui ne se réduit jamais entièrement à ses déterminations sociales.

Des processus psychiques et cognitifs sont en jeu dans les croyances en la légitimité d'un ordre social quelconque, d'une personne ou d'une institution. Réfléchir à la légitimité est une manière de réfléchir sur les conditions même de l'action. Une action est une suite coordonnée de mouvements visant à obtenir une transformation du monde. D'un

1 Nous renvoyons àl'article d'André Berten, « Légitimité, légitimation et normativité », dans ce volume.

2 On peut bien sûr entendre l'idée de justification au sens de L. BOLTANSKI et L. THEVENOT. Les «économies de la grandeur» visent à montrer que les classifications selon des « ordres » de grandeur ne sont pas dénuées de justification. «C'est à des actes justifiables que nous nous intéresserons, en tirant toutes les conséquences du fait que les personnes sont confrontées à la nécessité d'avoir à justifier leurs actions, c'est-à-dire non pas à inventer, après coup, de fausses raisons pour maquiller des motifs secrets, comme on trouve un alibi, mais à les accomplir de façon à ce qu'elles puissent se soumettre à une épreuve de justification », (De la justification. Les économies de la grandeur, Paris, Gallimard, 1991, 54-55). 
point de vue d'une théorie de l'action ${ }^{1}$, nous suivons constamment des « règles » auxquelles nous obéissons spontanément parce qu'elles sont les conditions de succès de l'action. Cela fait apparaître que le concept de normativité est implanté dans l'activité humaine avant d'être moral. Mais ce qui permet aux humains de considérer les normes en tant que normes et de pouvoir en interroger les contenus, c'est leur capacité réflexive. Cette capacité est liée à l'utilisation du langage. Ceci rend la question de la normativité plus complexe encore. Disons brièvement que ce que montrent des recherches de psychologues cognitivistes ${ }^{2}$ et de psychologues sociaux ${ }^{3}$, c'est que «notre connaissance du réel procède de catégorisations qui sont absolument nécessaires pour que le monde soit minimalement compris et utilisé». ${ }^{4}$ Dans la manière de présenter nos catégorisations, nos termes, nos concepts, des valeurs, des coutumes, des normes sont déjà présentes. Nous ne pouvons pas vivre sans nous soumettre à une certaine normativité. Bien que nous nous y conformions le plus souvent sans nous en rendre compte, les normes peuvent, réflexivement, faire l'objet de discussions et de mises en question. Elles ont une importance essentielle dans nos interactions. Les normes partagées auxquelles les individus se soumettent dans la vie sociale s'apparentent aux contraintes imposées par la vie en société.

La prise en considération de la normativité dans l'intersubjectivité et dans les relations sociales conduit à l'idée d'un «ordre normatif » essentiel au comportement de chaque individu et qu'il partage déjà toujours avec un certain nombre de ses semblables. L'ordre normatif provient de ce que Habermas considère comme le "monde vécu ${ }^{5}$ c'est-à-dire l'ensemble des ressources de sens partagées par les acteurs, le résultat de la socialisation. L'ordre normatif est un ordre social. Il n'est évidemment pas indépendant des déterminations sociologiques de la perception et de la faculté de jugement des individus mais il ne s'y

1 Nous renvoyons principalement aux travaux de E. ANSCOMBES, D. DAVIDSON, P. RICOEUR.

2 Cf. par exemple les travaux de M. JHONSON-LAIRD.

3 Cf. par exemple les travaux de D. KAHNEMAN et A. TVERSKI

4 BERTEN A., op.cit., p.81.

5 Rappelons cette distinction cardinale dans la théorie sociale de Habermas, entre système et monde vécu. Appartiennent au système, le sous-système de l'économie, ou du marché, régi par le medium de l'argent, et le sous-système du politique, ou de l'Etat et de l'administration, régi par le medium du pouvoir. Quant au monde vécu, il englobe à la fois la société civile et les activités proprement privées. 
réduit pas. Le rapport de chaque individu à l'ordre normatif dépend intimement de ses capacités langagières et réflexives. Nous n'avons pas tous les mêmes raisons d'obéir à l'ordre normatif.

Revenons à la question de la légitimité. On peut penser que les justifications avancées par le pouvoir seront acceptées ou refusées par les acteurs selon qu'elles sont conciliables ou non avec l'ordre normatif auxquelles ils se réfèrent. Une approche normative permet de mieux saisir comment le processus de légitimation intervient dans la relation entre pouvoir et monde vécu. Comme le souligne Habermas, la relation de pouvoir se caractérise par un déséquilibre structurel des satisfactions immédiates attendues par les parties. A la différence d'une transaction commerciale, par exemple, où chaque partie reçoit en principe une contrepartie équivalente à ce qu'elle a cédé, dans le rapport de pouvoir, le consentement est accordé par l'individu sans autre contrepartie. La «compensation» de cette perte ou de ce sacrifice est sans doute la reconnaissance d'une utilité collective. Or, « comme celui qui a le pouvoir use de son pouvoir de définition et fixe les buts à faire valoir collectivement, un rééquilibrage de l'infériorité structurelle ne pourra se réaliser que si les sujets soumis au pouvoir peuvent mettre à l'épreuve, d'un point de vue normatif, les buts proposés eux-mêmes et s'ils peuvent ou les confirmer ou les rejeter: ils doivent pouvoir contester que les buts fixés soient souhaitables ou qu'ils soient, comme nous disons, dans l'intérêt général ${ }^{1}$. En d'autres termes, étant donné que le pouvoir ne peut fournir dans l'interaction elle-même un équivalent comme le fait l'argent dans l'échange, c'est l'ordre normatif qui doit fournir des raisons d'obéir. "Dans les rapports de pouvoir, conclut Habermas, seule la référence à des buts collectifs susceptibles de légitimation rétablit l'équilibre présent d'emblée dans la relation d'échange idéal-typique $»^{2}$.

Si l'on suit Habermas, la référence à l'intérêt général est idéalement la source de la légitimité. Mais cette référence ne prend valeur d'étayer la légitimité qu'à la condition de rencontrer des raisons d'obéir dans l'ordre normatif. En d'autres termes, les justifications du pouvoir

1 HABERMAS J., Théorie de l'agir communicationnel, Tome II : Pour une critique de la raison fonctionnaliste, Paris, Fayard,1987, p. 299.

2 HABERMAS J., Théorie de l'agir communicationnel, Tome II, p. 299. Ce que Habermas appelle une relation d'échange idéal-typique est la relation monétaire. 
doivent donc être recevables du point de vue de Quiconque dans le monde vécu des acteurs.

En se référant aux travaux du psychologue social pragmaticien Georges Herbert Mead ${ }^{1}$, on peut montrer que la présence de Neuter, point de vue «valant pour tous », est inhérente à l'ordre normatif. Mead a, en effet, démontré que dans une interaction aux configurations normées (et non aux configurations ajustées), les normes sont en position d'extériorité par rapport aux interactions. Cette position d'extériorité n'est pas une position de transcendance, elle ne suppose pas l'égalité des partenaires de l'interaction. Elle dépend des contraintes pragmatiques d'une interaction normée et constitue de ce fait un point de vue qui vaut pour tous. Ce n'est même qu'en idéalisant le rôle pragmatique de Neuter que la normativité émerge ${ }^{2}$.

On peut faire l'hypothèse que cette observation permet de concevoir des modes de légitimation ou de re-légitimation susceptibles d'échapper au piège de l'idéologie ou de la tromperie, en sortant d'une version purement symétrique ou ajustée des interactions pour faire droit au cœur du lien social à la constitution d'un point de vue « valant pour tous », Neuter, fondant une obligation comme obligation. «Les justifications données tant à la norme qu' au pouvoir dépendent certes d'interactions entre agents compétents. Cependant en tant qu'elles fondent des obligations (et pas seulement des conventions et des commandements), elles dépendent d'un ordre «externalisé » »³. Là où les idéologies visent à susciter le consentement des individus en jouant sur les fondements affectifs de leur rapport à la norme (croyance, désir, crainte, intérêt, etc.), un processus de légitimation peut ou pourrait, au contraire,

1 Il faut se rappeler la féconde distinction introduite par G. Mead entre play (jeu libre) et game (jeu réglementé) et l'importance de distinguer dans une interaction les configurations ajustées et les configurations normées. De simples ajustements interactifs en situation n'ont rien de vraiment normatif. Leur contingence n'implique pas un «engagement moral», c'est-à-dire la conscience d'une obligatoriété valable pour toute personne se trouvant dans la même situation. (MEAD G., L'esprit, le Soi et la société, Paris, PUF, 1963).

2 L'idéalisation sémantique permet de généraliser des attentes réciproques de comportement et cela éventuellement entre de multiples acteurs, sans pour autant acquérir le statut de norme.

3 DE MUNCK J, L'institution sociale de l'esprit, Paris, L'interrogation philosophique, PUF, 1999,p. 183. 
prendre appui sur leurs capacités communicationnelles, réflexives et délibératives.

Revenons maintenant à la médiation institutionnelle et aux conditions de sa mise en oeuvre. Nous avons vu qu'elle répondait à une tentative de solutionner un problème de légitimation, de façon régulée et systématique. La légitimation dont il s'agit ne concerne pas le pouvoir en général mais bien le pouvoir de l'Etat et l'action publique.

\section{La légitimation par le bas}

Les questions normatives sont au cœur des pratiques de médiation institutionnelle. Quand les administrés font appel au médiateur institutionnel afin qu'il intervienne «en tiers » dans la résolution de leurs conflits, dans la facilitation de leurs communications ou dans le traitement de leurs plaintes, on peut dire que la référence à un ordre normatif partagé est en «crise». Il y a un déficit de légitimité. Comment le médiateur institutionnel va-t-il intervenir en tiers ? Même si par certains aspects il en est proche, il n'est ni un juge, ni un arbitre. La parole du médiateur n'est dotée d'aucune force judiciaire et le médiateur ne tranche pas les conflits.

\section{Un cadre contraignant en mutation}

Une spécificité des conflits entre les administrés et l'Etat est qu'ils se déroulent dans un cadre législatif contraignant et qu'ils se règlent le plus souvent par des décisions unilatérales. Or, ce mode de fonctionnement ne satisfait plus aux exigences d'efficacité et de légitimité de nos sociétés et c'est bien pour cela que les instances de médiation ont été mises en place. C'est ce que soulignent les médiateurs :

La relation entre citoyen et autorité dépasse désormais le cadre juridique. D'une part, le citoyen n'est plus seulement justiciable, mais participant actif au pouvoir administratif. Il veut un bon service et est devenu exigeant. D'autre part, l'administration a subi des changements. Elle offre aujourd'hui un large éventail de services et elle est censée réfléchir à l'accomplissement de ses tâches. Cette dimension de professionnalisme requiert 
parfois une analyse allant au-delà de la seule application des règlements. ${ }^{1}$

Les médiateurs se heurtent à une sorte de carcan légal ou juridique dans leurs pratiques quotidiennes.

Lorsque l'administration applique la loi qu'elle doit appliquer mais qu'une situation inacceptable résulte de la décision prise, nous n'avons aucun pouvoir de contrainte sur l'administration, nous sommes heurtés dans notre sentiment de justice. Ce qui est heurtant, c'est que tous les cas soient traités de la même manière. Il n'est pas rare que l'on nous dise : «mais de quoi vous mêlez-vous ? ».

Ils s'accordent pour dire que, une fois franchi la cap d'une méfiance légitime, des relations de collaboration se sont progressivement mises en place avec les agents et services de l'administration.

La collaboration avec le monde politique est complexe, parfois très difficile ${ }^{2}$. Si certains responsables politiques soutiennent indéfectiblement l'institution de la médiation, d'autres sont très méfiants. A propos des réticences, plusieurs hypothèses sont évoquées : l'autorité du médiateur (que pourtant ils instituent) serait ressentie comme une source de concurrence, les freins seraient liés à une crainte vis-à-vis d'un organe de contrôle externe, indépendant du gouvernement comme du Parlement et finalement à une question de culture politique. Dans leurs rapports annuels, par exemple, de nombreux médiateurs tirent, avec élégance, la sonnette d'alarme. Les recommandations générales qu'ils adressent chaque année au Parlement et au gouvernement ne sont pas, ou pas suffisamment, suivies d'effets.

Depuis cinq années, nous insistons pour que nos rapports fassent l'objet d'une discussion au sein des Commissions parlementaires

1 MONETTE P.-Y., «De la légitimité de l'Ombudsman» in Ombudsman : quelles valeurs au cœur de la fonction?, Rapport du séminaire du 23 octobre 2001, Bruxelles, Ed. de la Fondation Roi Baudouin, 2002.

2 On se souvient des entraves politiques qui ont bloqué la réélection des médiateurs fédéraux pendant plus de cinq années. Cf. la presse belge entre janvier 2005 et octobre 2005. Cf. MONETTE P.-Y., « L'incroyable sage autour des médiateurs fédéraux », in Belgique où vas-tu? Entretiens avec Christian Laporte, Bruxelles, Ed. Mardaga, 2007. 
compétentes. Cette année, il semble qu'un écho soit donné à notre invite. Nous la réitérons toutefois dans ce sixième rapport annuel. ${ }^{1}$

La médiation institutionnelle est traversée par des logiques d'action très contradictoires. Nous l'avons vu plus haut, la nouvelle mission d'efficacité et de légitimité de l'administration publique la plonge dans un champ de contradictions et de conflits. Les fonctionnaires, amenés à rentrer en contact avec un nombre croissant de partenaires extérieurs, doivent repenser leur rapport avec le public. Différents discours circulent et formatent des pratiques distinctes. Sous l'effet des logiques managériales, un discours abordant le public comme un ensemble de clients potentiels cherche à s'imposer. L'administré est traité comme un «client » à servir et à satisfaire. Mais une administration publique n'est pas une entreprise. Un second discours consiste à traiter le public comme un ensemble de «citoyens». L'administré est alors traité comme un « citoyen » à qui on donne la parole et qui participe à la prise de décisions qui le concerne. Sur le terrain, ces deux discours n'apparaissent pas aussi distinctement, ils se mélangent. Le discours le plus fréquent est sans doute une sorte de compromis entre eux : un discours fonctionnaliste. On parle de l'administré comme d'un « usager». Ces différents discours mettent bien en évidence une pluralité de régimes de légitimité.

Dans sa pratique quotidienne, le médiateur privilégie tantôt l'une, tantôt l'autre version de son métier de constructeur de consensus. Se tient-il sur le versant du service et sur celui de la citoyenneté ? Inscrit-il ses interventions dans un discours managérial, citoyen ou fonctionnaliste ? Répondre à ces questions n'est pas facile. Et tous les choix sont conflictuels. S'il abonde, par exemple, dans le sens du « service » et du « client », il peut potentiellement perdre de vue sa place démocratique.

\section{Des principes}

La spécificité du médiateur institutionnel tient à ce que ce sont des principes qui guident son action. Le médiateur est saisi de plaintes et de réclamations introduites par des administrés. Ceux-ci ressentent que leurs droits ne sont pas respectés, qu'une injustice leur est faite, qu'il

1 HANNESSE J.-M. et G. SCHUERMANS, Rapport annuel 2004, Service médiation pour les Pensions, p. 2. 
y a dysfonctionnement dans l'application d'une loi ou d'une mesure administrative qui les concerne. En termes habermassiens, on dira que la situation rend manifeste un point de disjonction entre le monde vécu des acteurs et le système.

Face à cette situation, certains principes guident l'action du médiateur institutionnel : le principe d'écoute, de respect, du raisonnable, de courtoisie, de gestion consciencieuse, de bonne administration ${ }^{1}$, d'équité2.

Guidé par ces principes, le médiateur commence par s'assurer de la loi (lois, arrêtés, directives, règlements etc.). Dans certains cas, son travail consiste à faire apparaître qu'une plainte parfois confuse recouvre un rapport à un droit existant.

Mais la prise en compte de la loi et des principes ne fait pas encore la spécificité de la médiation dans la fonction publique. Comme nous l'avons vu plus haut, la question des normes est importante. En deçà de la loi et des principes, on trouve des normes constitutives de relations sociales et de culture organisationnelle. La revendication des droits et leur application s'inscrivent dans la culture normative de l'administration publique qui les met en œuvre. Le médiateur institutionnel, dans sa pratique quotidienne, est directement confronté au fait qu'un droit n'a pas la même signification pour tous les acteurs. Leur mission est d'écouter ce qui se vit dans le «monde vécu » des acteurs, de rencontrer les fonctionnaires, de comprendre les exigences d'efficacité et les normes locales.

La médiation institutionnelle répond à une demande sociale de justice au singulier. En ouvrant un espace d'écoute, d'altérité et de discussion, le médiateur met en place un dispositif de justice de "reconnaissance $»^{3}$. Le besoin de reconnaissance s'exprime chez les

1 Le principe de bonne administration suppose qu'une administration ne se réduit pas à des pratiques fonctionnelles mais vise un Bien Commun qui l'oblige à aller au-delà d'elle-même.

2 L'application de l'équité concerne les cas pour lesquels la décision prise par l'administration, bien que parfaitement légale et respectant les principes de la bonne administration, porte gravement atteinte au sentiment naturel de justice humaine et pour lesquels le médiateur, au nom de l'équité, invite l'administration à modifier sa décision.

3 Nancy FRASER distingue l'idée d'une justice de « distribution » des biens d'une idée de justice de « reconnaissance ». La lutte pour la reconnaissance, écrit-elle, est rapidement occupée à devenir la forme paradigmatique du conflit politique de cette fin de siècle. Qu'est-ce que la justice sociale? Reconnaissance et distribution, édition établie, traduction et introduction par Estelle Ferrarese, Paris, Editions la Découverte, coll. « Textes à l'appui/politique et sociétés », 2005. 
administrés par le besoin d'être écouté, de ne pas être traité comme un dossier, de ne pas être pris dans des catégories préétablies, de ne pas être sérié. La mise en place au niveau institutionnel d'un tel dispositif constitue en soit un facteur de légitimation de l'action publique. Le médiateur relégitimise l'action publique par le bas en reconnectant, selon la terminologie habermassienne, le «monde vécu des acteurs » et le « système ».

\section{Un tiers réflexif et délibératif}

Dans la recherche de consensus, le médiateur intervient en tiers neutre, indépendant et impartial. La notion de la tiercéité est centrale dans la médiation. Alors que la négociation peut se passer de la présence d'un tiers, celle-ci fonde le processus de médiation. En général, l'idée de tiers vise une personne tierce. Pourtant, pour comprendre ce qui se joue dans une médiation, il faut prendre un certain recul par rapport à cette conception. On ne peut réduire la question du tiers à une personne, à une tierce personne. Le concept de tiers implique une dimension d'extériorité. Intervenir en tiers peut signifier activer ou réactiver du tiers dans une interaction. Ce qui fait tiers peut donc avoir une existence distincte d'une personne tierce.

Nous avons montré qu' une approche pragmatique ${ }^{1}$ permet de penser le tiers comme une contrainte pragmatique c'est-à-dire une contrainte portant davantage sur les manières d'agir que sur les contenus d'action. Ainsi, ce que Mead nomme Neuter - point de vue «valant pour tous » ou Autrui généralisé - fait fonction de tiers dans une interaction normée. Cette forme de tiers, nous avons proposé de l'appeler tiers généralisé.

Quand les administrés s'adressent au médiateur, ce qui fait tiers généralisé - la référence à un point de vue qui vaut pour tous, à un ordre normatif partagé - est en crise. Comment le médiateur va-t-il intervenir pour créer du consensus ? Il peut chercher à construire un accord autour d'un ordre normatif qu'il pose en tiers. Mais ce n'est pas là que se situe

1 Cf. E. VOLCKRICK, « La question du tiers dans les dispositifs de médiation », in Avonsnous encore besoin d'un tiers?, LEBRUN J-P. et E. VOLCKRICK (éds), Toulouse, érès, coll. «Humus», 2005, p. 133-158 et «Intervenir en tiers aujourd'hui », in Négociations, De Boeck Université, Bruxelles n 1, 2007, p.75-88. 
l'essentiel de sa fonction. Pour bien saisir ce qui se joue là, nous devons contextualiser le registre de l'ordre normatif et du tiers généralisé.

Effectivement, les situations complexes et contradictoires auxquelles sont confrontés les médiateurs institutionnels relèvent de plusieurs «mondes » et chacun de ces mondes possède une normativité propre. On peut considérer qu'il n’y a pas un tiers généralisé mais des tiers généralisés. Il n'y a pas un ordre normatif mais des ordres normatifs. C'est ici sans doute que se passe quelque chose de spécifique à notre époque. Les normes partagées n'étant plus garanties par les évidences d'un monde commun, il faut désormais les expliquer, les justifier, en discuter le contenu.

Comme nous l'avons indiqué, cette contextualisation de la norme appelle un accompagnement : un médiateur. Le médiateur met à la discussion le contenu des normes à partir de principes. Il active alors une autre forme de tiers : un tiers réflexif et délibératif. La justification des normes ne pouvant se faire que par le langage, les contraintes dont il est question ici sont des contraintes communicationnelles, c'est-à-dire à des principes dont la validité se maintient au-delà de la diversité des normes. Comme le souligne Berten, «c'est la force de l'argument de Habermas, de ne pas renvoyer la question de la légitimité aux propriétés psychologiques des sujets, à leur tendance à attribuer une autorité à des personnes ou des institutions, et donc de ne pas se fonder sur la bonne volonté des individus, mais bien sur les contraintes du discours communicatif $» .1$

\section{Une autorité sans pouvoir}

Ces différentes caractéristiques montrent combien l'action du médiateur institutionnel est impossible sans qu'il ne dispose d'une autorité. Son autorité peut paraître bien mince par rapport à celle d'un juge ou même d'un arbitre et pourtant elle est indéniable. C'est une «autorité sans pouvoir $»^{2}$. Le pouvoir est l'ensemble des moyens de

1 A. BERTEN, «Légitimité, légitimation et normativité », dans ce volume, p. 77

2 DE MUNCK J., " Plus qu'un conseil et moins qu'un ordre », Chroniques de Droit Public, Publiekrechetijke Kronieken, Dossier: Pouvoir politique et médiation institutionnelle, De relatie tussen politiek en de ombudsman, $\mathrm{n}^{\circ} 2$, 2006. p. 525530. 
contrainte, des mesures, des ordres, des commandements, des décisions. Il y a toujours inévitablement de la violence dans le pouvoir. L'autorité est différente. L'autorité n'a pas de moyens de contrainte. On fait autorité. Hanna Arendt ${ }^{1}$ cite la définition qu'en donne le grand romaniste Theodor Momssen : "plus qu'un conseil et moins qu'un ordre, un avis auquel on ne peut passer outre sans dommage ». Les médiateurs se retrouvent particulièrement bien dans cette définition. Ils se servent de l'écrit mais leur principal instrument de travail est la parole : « une parole qui fait autorité sans être autoritaire, un conseil dénué de tout recours à la force. La présence du médiateur dans le système de la décision administrative, c'est le rêve moderne d'une autorité de la parole dans un monde qui en a perdu le sens $\gg .^{2}$

La médiation institutionnelle n'est pas un gadget communicationnel de plus qui mettrait de l'huile dans des rouages grippés, légitimerait la décision politique ou qui, comme on l'entend parfois, noierait dans le consensus les conflits susceptibles de questionner les fondements d'un capitalisme triomphant. Elle témoigne de mutations importantes dans les rapports entre administration, citoyenneté, justice et démocratie.

La médiation institutionnelle est un dispositif de régulation qui intègre réflexivement la communication comme une condition de son fonctionnement. Sa visée de création de consensus par le bas en fait un mode de légitimation nouveau : c'est avec la source même de la légitimité démocratique que communique le médiateur. C'est dans la mesure où le médiateur peut occuper sa place d'autorité réflexive que la médiation institutionnelle exerce une fonction de régulation et de légitimation de l'action de l'Etat et contribue au renouvellement de la démocratie.

1 ARENDT H., «Qu'est-ce que l'autorité ? », in La crise de la culture, trad. franç. 1972, Paris, Gallimard, Folio-Essais, p. 162.

2 DE MUNCK J., Ididem, p. 529. 